\title{
Hypertension in Patients with Chronic Kidney Disease
}

\author{
${ }^{1} \mathrm{P}$ Vijay Varma, ${ }^{2} \mathrm{M}$ Rajasekara Chakravarthi, ${ }^{3} \mathrm{G}$ Jyothsna
}

\begin{abstract}
The prevalence of hypertension in chronic kidney disease (CKD) patients exceeds that of the general population. Uncontrolled hypertension plays a significant role in progression to end stage renal disease and results in increased cardiovascular morbidity and mortality. A complex interplay between various pathophysiologic mechanisms is responsible for the development of hypertension in this patient population. The major factors being extracellular volume overload, increased endothelin-1 release and excess renin-angiotensin-aldosterone system and sympathetic nervous system activity. Dietary and lifestyle modifications have synergistic effects to drug therapy in the control of hypertension. There is no single blood pressure target that is optimal for all CKD patients. It is important to individualize the treatment depending on age, the severity of albuminuria, and comorbidities. Drugs blocking the renin-angiotensin-aldosterone system are the recommended first-line antihypertensive agents for most CKD patients. Intradialytic hypertension may be prevented by individualizing the dialysis prescription and using nondialyzable antihypertensives. New onset of hypertension in the elderly or new onset of difficult to control hypertension in a previously well controlled hypertensive patient should prompt the work up for atherosclerotic renal vascular disease.
\end{abstract}

Keywords: Aldosterone, Angiotensin, Chronic, Hypertension, Kidney, Renin.

How to cite this article: Varma PV, Chakravarthi MR, Jyothsna G. Hypertension in Patients with Chronic Kidney Disease. Hypertens J 2016;2(1):28-34.

Source of support: Nil

Conflict of interest: None

\section{INTRODUCTION AND EPIDEMIOLOGY}

Hypertension is an important cause of chronic kidney disease (CKD) and plays a significant role in its progression. On the contrary, hypertension is highly prevalent in CKD patients, which, if uncontrolled, results in high cardiovascular morbidity and mortality of this particular population. Data from the Indian Screening and Early Evaluation of Kidney Disease (SEEK) study show that prevalence of hypertension and CKD in the SEEK cohort is 43.5 and $17.2 \%$ respectively, and that the prevalence of hypertension in patients with CKD exceeds that of the general population $(64.5 \%$ in CKD

\footnotetext{
${ }^{1-3}$ Consultant

${ }^{1-3}$ Department of Nephrology, Star Kidney Center, Hyderabad Telangana, India
}

Corresponding Author: M Rajasekara Chakravarthi, Consultant, Department of Nephrology, Star Kidney Center Hyderabad 500034, Telangana, India, Phone: 09391059322 e-mail: rajasekarac@gmail.com population $v s 41.9 \%$ in non-CKD population). ${ }^{1}$ The prevalence of hypertension is elevated in patients with kidney damage and a normal glomerular filtration rate, and increases further as the glomerular filtration rate falls. Data from the modification of diet in renal disease study, e.g., showed that the prevalence of hypertension rose progressively from 65 to $95 \%$, as the glomerular filtration rate fell from 85 to $15 \mathrm{ml} / \mathrm{min}$ per $1.73 \mathrm{~m}^{2}{ }^{2}$

\section{PATHOPHYSIOLOGY OF HYPERTENSION IN CHRONIC KIDNEY DISEASE}

Although the kidney is involved in essential or primary hypertension, its insufficiency causes high blood pressure (BP), contributing to 2 to $5 \%$ of all cases of hypertension or half the cases of all forms of secondary hypertension. ${ }^{3}$ The pathogenesis of hypertension related to CKD is complex and multifactorial, mainly in the late stages of the renal disease (Table 1).

\section{Sodium and Volume Status}

Hypertension in CKD is related to both increases in cardiac output and increased peripheral vascular resistance (Flow Chart 1). Positive sodium balance can affect either component and achieving sodium balance remains a primary target of managing hypertension in patients with CKD. Although alteration of either cardiac output or vascular resistance would initially be expected to affect BP in a concordant direction, a healthy kidney can adapt to shortterm changes in BP to restore normotension. An increase in renal sodium excretion is the expected response to increased BP in healthy individuals. This pressure natriuresis allows for the restoration of extracellular volume and BP following an increase in cardiac output. However, in $\mathrm{CKD}$, this homeostatic mechanism is impaired, and the

Table 1: Pathophysiologic mechanisms of hypertension in CKD
1. Extracellular volume overload
2. Excess renin-angiotensin-aldosterone system activity
3. Excess sympathetic nervous system activity
4. Increased endothelin-1 release
5. Decreased activity of renal dopaminergic system
6. Reduced renalase levels
7. Oxidative stress
8. High arginine vasopressin levels
9. Secondary hyperparathyroidism
10. Drugs: Erythropoietin
11. High levels of endogenous digitalis-like factors (EDLFs) 
Flow Chart 1: Interplay between various pathophysiologic mechanisms causing hypertension in CKD

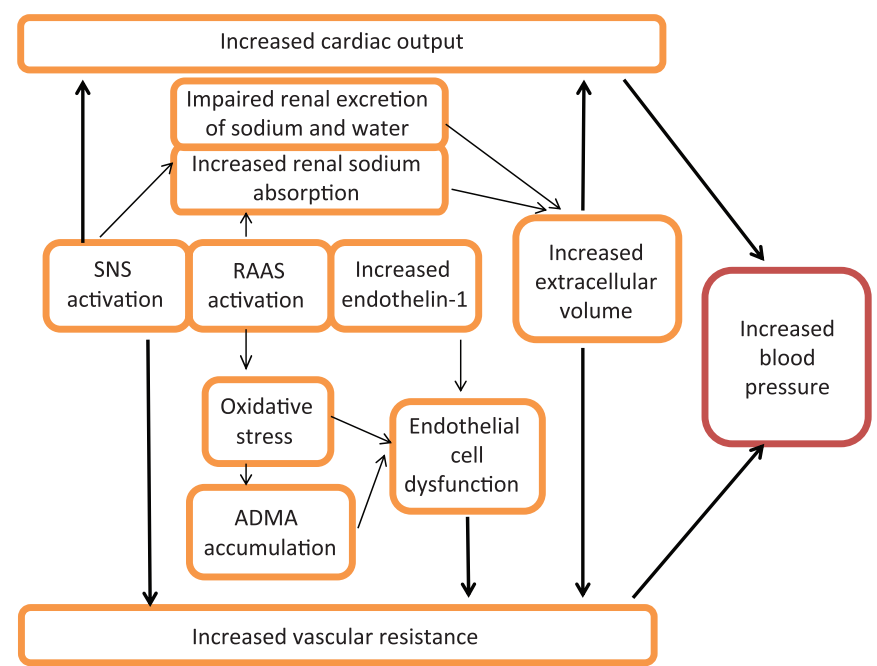

kidney fails to sufficiently excrete sodium loads. In CKD and end-stage renal disease (ESRD), it is hypothesized that an increase in cardiac output initiates the increase in BP, but ultimately, an increase in vascular resistance sustains the BP elevation. ${ }^{4,5}$ Accordingly, addressing both volume status and the degree of peripheral vasoconstriction are necessary to control BP in these patient populations. The effects on BP related to sodium intake ("salt sensitivity") are augmented with the progression of renal disease. ${ }^{6}$

\section{Role of Renin-Angiotensin-Aldosterone System}

The renin-angiotensin-aldosterone system (RAAS) has local and systemic effects that control BP by altering renal sodium reabsorption and vascular resistance. Renin released from juxtaglomerular cells cleaves angiotensinogen to Angiotensin I (Ang I), which is ultimately converted into Angiotensin II (Ang II) by the angiotensin-converting enzyme (ACE). Angiotensin II causes vasoconstriction upon binding to angiotensin type 1 (AT1) receptors in vascular smooth muscle cells (VSMCs). Angiotensin II increases proximal tubular reabsorption of sodium and stimulates aldosterone release from the adrenal gland, which is responsible for further sodium reabsorption in the distal nephron. Although this proposed sequence applies to the levels and activity of RAAS components in the plasma, there is also local RAAS activity. In CKD, mainly secondary to vascular disease, diabetes, or polycystic kidney disease, in areas of renal injury or ischemia, there is a greater production of local and intrarenal Ang II, which then exacerbates systemic hypertension. ${ }^{7,8}$

\section{Role of Renin and Aldosterone}

Following the use of an ACE inhibitor or angiotensin receptor blocker (ARB), serum aldosterone levels typically decrease. However, in up to $40 \%$ of patients receiving these medications, aldosterone levels can rebound to pretreatment levels through a process referred to as "aldosterone escape." ${ }^{\prime 9}$ Aldosterone increases BP via enhanced sodium reabsorption in the distal nephron, but it is also likely involved in vasoconstriction via interaction with the Ang II receptor. ${ }^{10}$

In contrast to the expected decrease in aldosterone following ACE inhibitor or ARB use, renin levels and plasma renin activity (PRA) are expected to increase as a result of the blockade in events downstream from the main actions of renin. It has been demonstrated in vivo that Ang II can be generated by enzymes other than ACE. ${ }^{11}$ This presents a potential obstacle to complete RAAS blockade if renin levels are sufficiently elevated in the context of an ACE inhibitor or ARB administration, and the possible use of add-on therapy to ACE inhibitors or ARB with a direct renin inhibitor.

\section{Sympathetic Nervous System and Hypertension}

The sympathetic nervous system activity is hyperactive in CKD. The mechanisms responsible are thought to be related to an increased renal nerve activity. The kidney possesses baroreceptors and chemoreceptors that increase renal nerve firing secondary to pressure changes or metabolites produced in response to ischemia or uremia. Afferent signals from the diseased kidney are transmitted to the vasomotor control center in the brain increasing the $\mathrm{BP}^{12}$ Renal sympathetic nerve stimulation increases proximal tubular reabsorption of sodium and water. ${ }^{13}$ In addition, the systemic effects of the SNS include increased cardiac output and vasoconstriction. Also, ESRD patients have increased muscle sympathetic nerve activity (MSNA) related to increases in RAAS activation.

\section{Renal Dopaminergic System}

Dopamine has a natriuretic effect by inhibiting Na-KATPase in proximal tubular segments. Patients suffering from CKD have reduced urinary excretion of dopamine and decreased activity of the renal dopaminergic system, thereby decreasing the sodium excretion. ${ }^{14}$

\section{Renalase}

Renalase is an amine oxidase, secreted into the circulation by kidneys, which contributes to catecholamine degradation. Patients with ESRD on hemodialysis have nearly undetectable levels, supporting the role of adequate renal function to maintain its presence. ${ }^{15}$

\section{Oxidative Stress and Nitric Oxide Antagonism}

Oxidative stress is a state of imbalance between oxidants and antioxidants in favor of the oxidants, which is detrimental to health. ${ }^{16}$ In CKD, excess oxidant molecules 
may directly stimulate vascular contraction or reduce nitric oxide, resulting in hypertension. ${ }^{17}$ The endothelialderived nitric oxide that causes vasodilatation is inhibited by increased levels of asymmetric dimethylarginine (ADMA) in CKD. ${ }^{18}$

\section{Arginine Vasopressin}

Arginine vasopressin (AVP) is a peptide released from the hypothalamus in response to increases in plasma osmolarity. Receptors for vasopressin include the V1a, V1b, and V2 receptors. Traditionally, V1a receptors were believed to be responsible for vasoconstriction via VSMC, and V2 receptors responsible for water reabsorption through the insertion of aquaporin channels in the collecting duct of the nephron. In CKD patients, AVP levels are higher and increase in response to osmolarity changes with a greater slope than in controls. ${ }^{19}$

\section{Secondary Hyperparathyroidism}

An association between increased BP and elevated serum parathyroid hormone (PTH) levels has been demonstrated in CKD patients. This could be due to higher cytosolic calcium in the subjects with elevated PTH levels. ${ }^{20}$

\section{Drug Related: Erythropoietin-Stimulating Agents}

Proposed mechanisms of erythropoietin-stimulating agent (ESA)-induced hypertension include increased endothelin-1 (ET-1) release and increased sensitivity to Ang II and adrenergic stimuli. ${ }^{21}$

\section{Role of Endogenous Digitalis-like Factors}

Endogenous digitalis-like factors (EDLFs) are substances, produced in the adrenal gland or in the hypothalamus, that inhibit the Na-K-ATPase in cell membranes, and also have natriuretic and vasoconstrictor properties. ${ }^{22}$ The high levels of EDLFs in CKD, secondary to an increased sodium content and expanded blood volume, may also contribute to the hypertension in these patients. ${ }^{23}$

\section{MANAGEMENT OF HYPERTENSION IN CHRONIC KIDNEY DISEASE}

The major goals of controlling BP in CKD patients are to prevent the progression to ESRD and to reduce cardiovascular complications. Dietary and lifestyle modifications have synergistic effects to drug therapy and hence play a very important role. Patient education and regular counseling are pivotal in achieving the goals.

\section{Salt Restriction and Lifestyle Modifications}

Recent kidney disease improving global outcomes (KDIGO) guidelines recommend lowering salt intake to less than $90 \mathrm{mmol}(<2 \mathrm{~g})$ per day of sodium (corresponding to $5 \mathrm{~g}$ of sodiumchloride), unless contraindicated. ${ }^{24}$ The rationale of salt restriction is that it directly has an effect on $\mathrm{BP}$ reduction in CKD patients and also facilitates the antiproteinuric effects of RAAS-blocking drugs. ${ }^{25}$ Highsalt diet blunts kidney autoregulation, which exposes the glomerulus to higher filtration pressures.

Cigarette smoking can result in progression of CKD by worsening the hypertension through sympathetic nervous system activation and also by increasing the plasma endothelin levels. ${ }^{26}$ The oxidative stress due to smoking can produce endothelial dysfunction and may result in the increase of BP. It is very important to counsel the patients regarding the consequences of smoking on renal function and to ensure strict abstinence.

Weight loss in CKD patients may result in reduction of proteinuria and better BP control. It also improves the long-term cardiovascular outcomes. ${ }^{27}$

\section{Pharmacological Therapy}

Antihypertensive drugs become mandatory as renal function worsens and patients usually need two or more drugs for hypertension control. There is no single BP target that is optimal for all CKD patients. It is important to individualize the treatment depending on age, the severity of albuminuria, and comorbidities. In general, the available evidence indicates that in CKD patients without albuminuria, the target $\mathrm{BP}$ should be $\leq 140 \mathrm{~mm} \mathrm{Hg}$ systolic and $\leq 90 \mathrm{~mm} \mathrm{Hg}$ diastolic. However, in most patients with an albumin excretion rate of $\geq 30 \mathrm{mg} / 24 \mathrm{~h}$, a lower target of $\leq 130 \mathrm{~mm} \mathrm{Hg}$ systolic and $\leq 80 \mathrm{~mm} \mathrm{Hg}$ diastolic is suggested. ${ }^{28}$ It is important to keep in mind that lowering the $\mathrm{BP}<120 / 70 \mathrm{~mm} \mathrm{Hg}$, mainly in older patients, can increase the progression of renal failure and enhance the probability of a cardiovascular event.

\section{RAAS Blockade}

Drugs blocking the RAAS system are the recommended first-line antihypertensive agents for most CKD patients, with the most commonly used agents being ACE inhibitors or ARBs. These agents should be titrated to the maximum recommended doses in order to achieve the greatest RAAS inhibition. The benefits of ACE inhibitors and ARB in CKD are much beyond hypertension control. They reduce the intraglomerular hydrostatic pressure, proteinuria and also the levels of Ang II. ${ }^{29}$ Angiotensin II is an octapeptide with proinflammatory and profibrotic actions. It stimulates the production of transforming growth factor- $\beta$, which results in extracellular matrix deposition. As a result, these drugs also reduce cardiovascular morbidity and mortality. Patients must be closely followed up for hyperkalemia and for any rise in 
serum creatinine after initiation of these drugs or every time the drug dose is increased. A slight increase of the creatinine level ( $<25 \%$ from baseline) is acceptable, as it reflects a decrease of the glomerular hyperfiltration and is beneficial in the long run. A significant increase of the creatinine level ( $>25 \%$ from baseline) is suggestive of volume depletion (concomitant use of diuretics) or of bilateral renal artery stenosis. Treating physician must counsel the patient regarding the need to avoid potassium-rich foods. Other hyperkalemic drugs, such as aldosterone antagonists or nonsteroid anti-inflammatory drugs, need to be avoided.

Limesh et $\mathrm{al}^{30}$ devised a scoring system for RAAS blockade (RASB) wherein the amount of an ARB and ACE inhibitor was scored as the dose of that agent used in each patient divided by the maximum recommended antihypertensive dose (Table 2). For example, if a patient received $80 \mathrm{mg}$ of enalapril and $50 \mathrm{mg}$ of losartan simultaneously, RASB score in that patient would be $2.5(80 / 40+50 / 100$ or $2+0.5)$. The rate of change in estimated glomerular filtration rate (eGFR) was $-1.5 \pm 5.0$ $\mathrm{ml} / \mathrm{min} / 1.73 \mathrm{~m}^{2}$ per year in patients who received RASB $(\mathrm{N}=168)$ and $-6.0 \pm 5.4$ in those who did not $(\mathrm{N}=97)$ $(\mathrm{p}<0.001)$. In a subgroup of patients who received RASB, the incidence of decline of eGFR $>50 \%$ was $17.8 \%$ in the low-dose RASB group ( $\mathrm{N}=84$, RASB score $0.63 \pm 0.38$ ) and $4.8 \%$ in the high-dose group $(\mathrm{N}=84$, RASB score 2.5 $\pm 0.7)(p=0.001)$. RASB was associated with significantly better renoprotection in CKD of diverse etiology, even in nonproteinuric diseases. This effect appeared to be dose-dependent, with higher supramaximal doses exhibiting better renoprotection than the lower conventional doses.

\section{OTHER DRUGS}

Single antihypertensive drug might not be sufficient for BP control in most CKD patients. The choice of another antihypertensive agent should be individualized according to patient condition and comorbidities (Flow Chart 2).

\section{Diuretics}

Diuretics are crucial drugs in controlling hypertension, as volume overload contributes significantly to high BP

Table 2: Definition of maximum dose of renin-angiotensin system blocking agent

\footnotetext{
Maximum dose defined for individual $A R B$ agents were: Losartan 100 , valsartan 320 , telmisartan 80 , irbesartan 300 , and olmesartan $40 \mathrm{mg}$

Maximum dose defined for individual ACE inhibitor agents were: Enalapril 40, ramipril 20 and lisinopril $40 \mathrm{mg}$
}

Flow Chart 2: Algorithm for pharmacologic treatment of hypertension in CKD

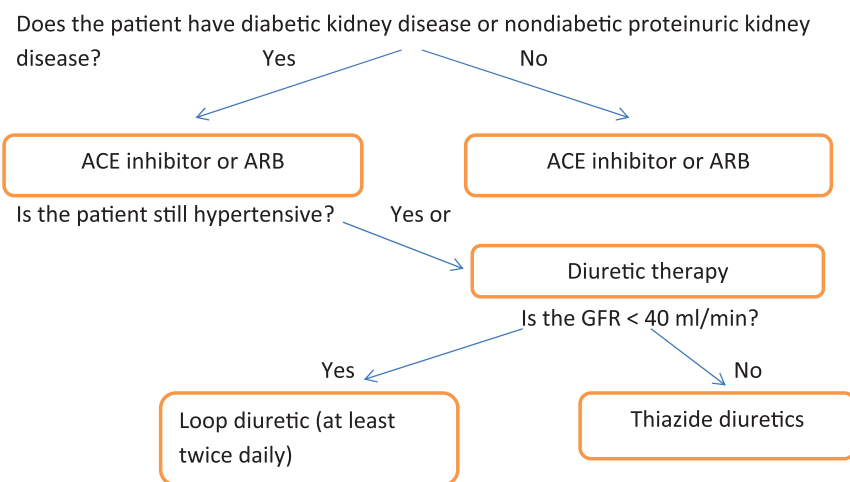

Is the patient still hypertensive?

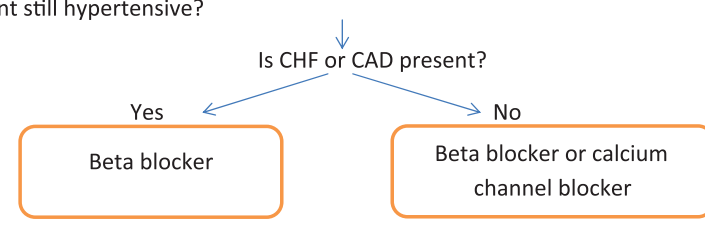

Is the patient still hypertensive?

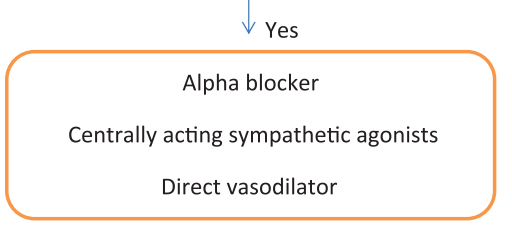

as CKD progresses. Thiazides are usually used when the GFR $>30 \mathrm{ml} / \mathrm{min} / 1.73 \mathrm{~m}^{2}$ body surface area, being ineffective beyond this level. Loop diuretics such as indapamide or furosemide may be useful in advanced stages of CKD. It is important to dose loop diuretics at least twice daily and to consider increased doses in patients with significantly impaired GFR in order to optimize delivery of the drug to the site of action. Diuretics diminish sodium reabsorption, thereby decreasing blood volume and hence stimulate renin production. Hence, the effect of ACE inhibitors and ARB is intensified when diuretics are added along with them. On the contrary, care must be taken not to overdose, as diuretics are known to cause dehydration (more common during summer), acute interstitial nephritis, and directly reduce the GFR by increased delivery of solute to the loop of Henle resulting in tubuloglomerular feedback. Thiazides can cause hyponatremia, which is an important cause of falls in elderly people. Potassium-sparing agents should be used carefully, particularly if the patient is on ACE inhibitor or ARB, as they may result in lifethreatening hyperkalemia. $^{31}$

\section{Dual Blockade of the Renin-Angiotensin- Aldosterone System}

Currently, there is no conclusive evidence favoring combined RAAS-blockade in diabetic or nondiabetic 
CKD. The sub-analysis of ONTARGET study reported an accelerated decline in estimated GFR (eGFR) and higher dialysis and mortality rates in a combined ARB and ACE inhibitor (ACE-I) therapy group. ${ }^{32}$ However, there was a definite improvement in BP and proteinuria. Because RAAS blockade in the ONTARGET study was not titrated against BP or proteinuria level, generalizing the study conclusions to other populations or to other indications for RAAS blockade therapy may be inappropriate.

\section{Calcium Channel Blockers}

Calcium channel blockers (CCBs) may be used as an add-on therapy if the patient has angina or heart failure secondary to diastolic dysfunction. ${ }^{31}$ Dihydropyridines such as amlodipine and nifidepine dilate only afferent arteriole, as they act only on L-type calcium channels, thereby resulting in glomerular hypertension, whereas cilnidipine also acts on $\mathrm{N}$-type calcium channels that are present in nerve terminals, resulting in dilation of both afferent and efferent arterioles and hence reduces glomerular pressure and thereby proteinuria. ${ }^{33}$ Hence, it is preferable to use cilnidipine in proteinuric CKD patients. Also, nondihydropyridine CCB may be added to an ACE inhibitor or ARB in patients with proteinuria. On the contrary, they should be avoided in patients with second and third-degree heart block or with congestive heart failure due to systolic dysfunction. ${ }^{31}$

\section{Beta-Adrenergic Blockers}

Chronic kidney disease is commonly associated with ischemic heart disease and hence beta-adrenergic blockers are particularly indicated in patients with prior angina or myocardial infarction or with atrial tachycardia or fibrillation. ${ }^{31}$ Atenolol and sotalol are water soluble and excreted by the kidney, and thus may accumulate in CKD, whereas the lipid-soluble ones (propranolol, metoprolol) are metabolized in the liver and may be associated with central nervous system side effects. Both types can cause hyperkalemia. Carvedilol is a beta-adrenergic blocker with nonselective $\alpha 1$ activity that has beneficial effects on lipid profile, has an antioxidant activity, and also reduces albumin excretion. ${ }^{34,35}$

\section{Aldosterone Antagonists}

The addition of aldosterone antagonists with Ang II antagonists may be justified by the so-called aldosterone escape phenomenon. ${ }^{9}$ The advantage of this add-on therapy is attributed to the antifibrotic and antihypertensive properties of aldosterone antagonists and there is also some reduction in proteinuria. However, the risk of hyperkalemia is increased.

\section{Renin Inhibitors}

Renin inhibitors block the conversion of angiotensinogen to Ang I. Aliskiren has been shown to reduce BP and albuminuria in type 2 diabetic patients, when given alone or in combination with losartan. ${ }^{36,37}$ The ALTITUDE study was carefully designed to compare the effects of combination treatment of aliskiren on top of ACE inhibitors or ARB versus ACE or ARB alone on cardiovascular and renal outcomes in 8561 patients with type 2 diabetes. The trial was prematurely terminated due to renal complications, hyperkalemia, and hypotension in the aliskiren group. ${ }^{38}$ Further studies are needed to determine whether aliskerin, alone or in combination, is able to reduce hard renal and cardiovascular end-points in CKD patients.

The $\alpha$-adrenergic blockers, centrally acting sympathetic agonists, or the potent vasodilator minoxidil are used only if BP control was still not achieved with the previous described classes of antihypertensive drugs. Minoxidil must always be associated with a betaadrenergic blocker and a diuretic in order to control the reflex tachycardia and the fluid retention induced by the former. $^{31}$

$\alpha$-adrenergic blockers may cause postural hypotension and should be used with caution.

\section{Intradialytic Hypertension}

There is no uniform definition of intradialytic hypertension (IDH). Intradialytic hypertension is defined as an increase in BP during or immediately after hemodialysis, which results in postdialysis hypertension. The frequency of IDH has been reported to be around 10 to $15 \%$ of the hemodialysis patients. ${ }^{39,40}$ In addition to the pathophysiologic factors mentioned in Table 1, certain other factors may be responsible for IDH. Dialysate-specific factors such as high sodium and high calcium dialysate or a low potassium dialysate have been proposed to cause IDH. Antihypertensive drug removal (particularly metoprolol and atenolol) during dialysis and high doses of erythropoietin result in IDH.

\section{Prevention of Intradialytic Hypertension}

Adequate care should be taken to make sure that patients are at their ideal dry weight. Routine withholding of antihypertensive medications prior to hemodialysis should be avoided. In addition, antihypertensive agents (carvedilol, labetalol, amlodipine, ARB, clonidine, hydralazine), which are not removed during dialysis, should be preferred. ${ }^{41}$ The dialysis prescription should be individualized to achieve adequate sodium solute removal. Routine use of high calcium dialysate should be avoided unless clinically indicated. The lowest possible dose of erythropoietin necessary should be used and 
preferably subcutaneous dosing rather than intravenous dosing should be considered.

\section{Hypertension in Chronic Kidney Disease Secondary to Atherosclerotic Renovascular Disease}

Chronic kidney disease that results from atherosclerotic renal artery stenosis is frequently called ischemic nephropathy. ${ }^{42}$ Findings that suggest the presence of renovascular disease include as follows:

- Severe hypertension that may be treatment resistant;

- An acute rise in serum creatinine following the administration of ACE inhibitors or ARB. This usually, but not invariably, resolves after withdrawal of the drug;

- Significant variability of serum creatinine concentration that may be due to changes in volume status (fluctuating renal parameters);

- Recurrent episodes of flash pulmonary edema and/ or refractory heart failure;

- Deterioration of renal function after placement of an endovascular aortic stent graft;

- New onset of hypertension in the elderly or new onset of difficult to control hypertension in a hypertensive patient who had a good control so far.

All patients should receive medical therapy to control their hypertension in addition to routine CKD care and surveillance. In addition to medical therapy and risk factor reduction, some but not all patients should undergo revascularization, depending upon the hemodynamic severity and likely recoverability of kidney function.

Results from studies of selected patients with ischemic nephropathy subjected to revascularization suggest that 25 to $30 \%$ will recover kidney function to a meaningful degree, sometimes avoiding progression to ESRD and/or the need for renal replacement therapy. Approximately $50 \%$ will have a little immediate change in kidney function but will "stabilize," whereas approximately $20 \%$ will have a progressive deterioration of kidney function, possibly related to the procedure. ${ }^{43}$

\section{CONCLUSION}

Hypertension is a prevalent comorbidity associated with CKD from the early stages to ESRD. A better understanding of the physiopathologic mechanisms is important to improve our treatment strategies. Irrespective of the etiology of CKD, controlling hypertension helps prevent its progression to ESRD. The target BP for CKD patients that optimally reduces the risk for both renal and cardiovascular outcomes remains unknown, but subjects with increased proteinuria derive more benefit from aggressive BP lowering. Salt restriction and lifestyle changes intensify the effects of pharmacologic antihypertensive agents, which will be required in most
CKD patients. Inhibitors of RAAS appear to be the most beneficial in CKD patients with proteinuria. Intradialytic hypertension may be prevented by individualizing the dialysis prescription and using nondialyzable antihypertensives.

\section{REFERENCES}

1. Singh AK, Farag YM, Mittal BV, Subramanian KK, Reddy SR, Acharya VN, Almeida AF, Channakeshavamurthy A, Ballal HS, P G, et al. Epidemiology and risk factors of chronic kidney disease in India: results from the SEEK (Screening and Early Evaluation of Kidney Disease) study. BMC Nephrol 2013 May 28;14:114.

2. Buckalew VM Jr, Berg RL, Wang SR, Porush JG, Rauch S, Schulman G. Prevalence of hypertension in 1,795 subjects with chronic renal disease: the modification of diet in renal disease study baseline cohort. Modification of diet in renal disease study group. Am J Kidney Dis 1996 Dec;28(6):811-821.

3. Kaplan NM. Treatment of hypertension: drug therapy. In: Kaplan NM, Flynn JT, editors. Clinical hypertension. 9th ed. Philadelphia: Lippincott Williams and Wilkins; 2006. p. 217-230.

4. Brod J, Bahlmann J, Cachovan M, Hubrich W, Pretschner PD. Mechanisms for the elevation of blood pressure in human renal disease. Preliminary report. Hypertension 1982 Nov-Dec;4(6):839-844.

5. Coleman TG, Bower JD, Langford HG, Guyton AC. Regulation of arterial pressure in the anephric state. Circulation 1970 Sep;42(3):509-514.

6. Koomans HA, Roos JC, Boer P, Geyskes GG, Mees EJ. Salt sensitivity of blood pressure in chronic renal failure. Evidence for renal control of body fluid distribution in man. Hypertension 1982 Mar-Apr;4(2):190-197.

7. Acosta JH. Hypertension in chronic renal disease. Kidney Int 1982;22(6):702-712.

8. Rosenberg ME, Smith LJ, Correa-Rotter R, Hostetter TH. The paradox of the renin-angiotensin system in chronic renal disease. Kidney Int 1994 Feb;45(2):403-410.

9. Bomback AS, Klemmer PJ. The incidence and implications of aldosterone breakthrough. Nat Clin Pract Nephrol 2007 Sep;3(9):486-492.

10. Yamada M, Kushibiki M, Osanai T, Tomita H, Okumura K. Vasoconstrictor effect of aldosterone via angiotensin II type 1 (AT1) receptor: possible role of AT1 receptor dimerization. Cardiovasc Res 2008 Jul 1;79(1):169-178.

11. McDonald JE, Padmanabhan N, Petrie MC, Hillier C, Connell JM, McMurray JJ. Vasoconstrictor effect of the angiotensinconverting enzyme-resistant, chymase-specific substrate [Pro(11)(D)-Ala(12)] angiotensin I in human dorsal hand veins: in vivo demonstration of non-ace production of angiotensin II in humans. Circulation 2001 Oct 9;104(15):18051808.

12. Rump LC, Amann K, Orth S, Ritz E. Sympathetic overactivity in renal disease: a window to understand progression and cardiovascular complications of uraemia? Nephrol Dial Transplant 2000 Nov;15(11):1735-1738.

13. Bell-Reuss E, Trevino DL, Gottschalk CW. Effect of renal sympathetic nerve stimulation on proximal water and sodium reabsorption. J Clin Invest 1976 Apr;57(4):1104-1107.

14. Casson IF, Lee MR, Brownjohn AM, Parsons FM, Davison AM, Will EJ, Clayden AD. Failure of renal dopamine response 
to salt loading in chronic renal disease. Br Med J (Clin Res Ed) 1983 Feb 12;286(6364):503-506

15. Xu J, Li G, Wang P, Velazquez H, Yao X, Li Y, Wu Y, Peixoto A, Crowley S, Desir GV. Renalase is a novel, soluble monoamine oxidase that regulates cardiac function and blood pressure. J Clin Invest 2005 May;115(5):1275-1280.

16. Sies H. Oxidative stress: oxidants and antioxidants. Exp Physiol 1997 Mar;82(2):291-295.

17. Hu Q, Corda S, Zweier JL, Capogrossi MC, Ziegelstein RC. Hydrogen peroxide induces intracellular calcium oscillations in human aortic endothelial cells. Circulation 1998 Jan 27;97(3):268-275

18. Vallance P, Leone A, Calver A, Collier J, Moncada S. Accumulation of an endogenous inhibitor of nitric oxide synthesis in chronic renal failure. Lancet 1992 Mar 7; 339(8793):572-575.

19. Argent N, Burrell L, Goodship T, Wilkinson R, Baylis P. Osmoregulation of thirst and vasopressin release in severe chronic renal failure. Kidney Int $1991 \mathrm{Feb}$;9(2): 395-400.

20. Raine AE, Bedford L, Simpson AW, Ashley CC, Brown R, Woodhead JS, Ledingham JG. Hyperparathyroidism, platelet intracellular free calcium and hypertension in chronic renal failure. Kidney Int 1993 Mar;43(3):700-705.

21. Kang DH, Yoon KI, Han DS. Acute effects of recombinant human erythropoietin on plasma levels of proendothelin-1 and endothelin-1 in haemodialysis patients. Nephrol Dial Transplant 1998 Nov;13(11):2877-2883.

22. Takahashi H, Yoshika M, Komiyama Y, Nishimura M. The central mechanism underlying hypertension: a review of the roles of sodium ions, epithelial sodium channels, the renin-angiotensin-aldosterone system, oxidative stress and endogenous digitalis in the brain. Hypertens Res 2011 Nov;34(11):1147-1160.

23. Komiyama $Y$, Dong $X H$, Nishimura N, Masaki H, Yoshika M, Masuda M, Takahashi H. A novel endogenous digitalis, telocinobufagin, exhibits elevated plasma levels in patients with terminal renal failure. Clin Biochem 2005 Jan;38(1): 36-45.

24. Kidney Disease: Improving Global Outcomes (KDIGO) Blood Pressure Work Group. KDIGO Clinical Practice Guideline for the Management of Blood Pressure in Chronic Kidney Disease. Kidney Inter Suppl 2012;2:337-414.

25. Vogt L, Waanders F, Boomsma F, de Zeeuw D, Navis G. Effects of dietary sodium and hydrochlorothiazide on the antiproteinuric efficacy of losartan. J Am Soc Nephrol 2008 May;19(5):999-1007.

26. Halimi JM, Mimran A. Renal effects of smoking: potential mechanisms and perspectives. Nephrol Dial Transplant 2000 Jul;15(7):938-940.

27. Praga M, Morales E. Weight loss and proteinuria. Contrib Nephrol 2006;151:221-229.

28. Wheeler DC, Becker GJ. Summary of KDIGO guideline. What do we really know about management of blood pressure in patients with chronic kidney disease? Kidney Int 2013 Mar; 83(3):377-383.
29. Sica DA. Combination angiotensin-converting enzyme inhibitor and angiotensin receptor blocker therapy: its role in clinical practice. J Clin Hypertens (Greenwich) 2003 NovDec;5(6):414-420.

30. Limesh M, Annigeri RA, Mani MK, Kowdle PC, Rao BS, Balasubramanian S, Seshadri R. Retarding the progression of chronic kidney disease with renin angiotensin system blockade. Ind J Nephrol 2012 Mar;22(2):108-115.

31. Kidney Disease Outcomes Quality Initiative. K/DOQI clinical practice guidelines on hypertension and antihypertensive agents in chronic kidney disease. Am J Kidney Dis 2004 May;43(5 Suppl 1):S1-S290.

32. Mann JF, Schmieder RE, McQueen M, Dyal L, Schumacher H, Pogue J, Wang X, Maggioni A, Budaj A, Chaithiraphan S, et al. Renal outcomes with telmisartan, ramipril, or both, in people at high vascular risk (the ONTARGET study): a multicentre, randomised, double-blind, controlled trial. Lancet 2008 Aug 16;372(9638):547-553.

33. Hayashi K, Wakino S, Sugano N, Ozawa Y, Homma K, Saruta T. Ca2+ channel subtypes and pharmacology in the kidney. Circ Res 2007 Feb 16;100(3):342-353.

34. Stafylas PC, Sarafidis PA. Carvedilol in hypertension treatment. Vasc Health Risk Manag 2008;4(1):23-30.

35. Bakris GL, Fonseca V, Katholi RE, McGill JB, Messerli FH, Phillips RA, Raskin P, Wright JT Jr, Oakes R, Lukas MA, et al. Metabolic effects of carvedilolvsmetoprolol in patients with type 2 diabetes mellitus and hypertension: a randomized controlled trial. JAMA 2004 Nov 10;292(18):2227-2236.

36. Persson F, Rossing $\mathrm{P}$, Reinhard $\mathrm{H}$, Juhl T, Stehouwer $\mathrm{CD}_{\text {, }}$ Schalkwijk C, Danser AH, Boomsma F, Frandsen E, Parving $\mathrm{HH}$. Renal effects of aliskiren compared with and in combination with irbesartan in patients with type 2 diabetes, hypertension, and albuminuria. Diabetes Care 2009 Oct;32(10):1873-1879.

37. Parving HH, Persson F, Lewis JB, Lewis EJ, Hollenberg NK, Investigators AS. Aliskiren combined with losartan in type 2 diabetes and nephropathy. N Engl J Med 2008 Jun 5;358(23):2433-2446.

38. Parving HH, Brenner BM, McMurray JJ, de Zeeuw D, Haffner SM, Solomon SD, Chaturvedi N, Persson F, Desai AS, Nicolaides M, et al. Cardiorenal end points in a trial of aliskiren for type 2 diabetes. N Engl J Med 2012 Dec 6;367(23):2204-2213.

39. Chazot C, Jean G. Intradialytic hypertension: it is time to act. Nephron Clin Pract 2010;115(3):c182-c188.

40. Inrig JK, Patel UD, Toto RD, Szczech LA. Association of blood pressure increases during hemodialysis with 2-year mortality in incident hemodialysis patients: a secondary analysis of the Dialysis Morbidity and Mortality Wave 2 Study. Am J Kidney Dis 2009 Nov; 54(5):881-890.

41. Inrig JK. Antihypertensive agents in hemodialysis patients: a current perspective. Semin Dial 2010 May-Jun;23(3):290-297.

42. Adamczak M, Wiecek A. Ischemic nephropathy: pathogenesis and treatment. Nefrologia 2012 Jul 17;32(4):432-438.

43. TextorSC,MisraS, Oderich GS. Percutaneous revascularization for ischemic nephropathy: the past, present, and future. Kidney Int 2013 Jan;83(1):28-40. 